\title{
COMBINED LEFT VENTRICULAR ANEURYSM AND THORACIC AORTIC PSEUDOANEURYSM CAUSED BY BLUNT CHEST TRAUMA
}

Ramón Adalia, MD, Luis Sabater, MD, Manuel Azqueta, MD, Xavier Muntanyá, MD, M. Isabel Real, MD, V. Riambau, MD, F. Xavier González, MD, and Elizabeth Zavala, MD, Barcelona, Spain

Trauma resulting from a fall is associated with multiple injuries, including a great variety of cardiac and vascular lesions occurring alone or in combination. These include cardiac disruption, aortic rupture, vessel injury related to bone fractures, and retroperitoneal hemorrhage associated with a high mortality rate. ${ }^{1}$ We present here an extremely rare and difficult to manage situation in a patient with multisystemic injury, with the combination of blunt cardiac injury and disruption of the aorta leading to a ventricular aneurysm and formation of an aortic pseudoaneurysm.

Clinical summary. A 20-year-old woman was brought to the emergency department of our hospital after a $15-\mathrm{m}$ fall. Initial evaluation on arrival showed an awake patient with a Glasgow Coma Scale score of 13, normal blood pressure, tachycardia, and marked hypoventilation of the left lung as a result of left thoracic contusion. The thoracic radiograph demonstrated multiple rib fractures of the left side of the chest with hemopneumothorax and without mediastinal widening. She also had a subcapital fracture of the left femur, pelvis, and transverse apophysis of the lumbar spine (L3, L4,

From the Surgical Intensive Care Unit, Department of Cardiology, Department of Angioradiology, Department of Cardiovascular Surgery, Hospital Clinic, University of Barcelona, Barcelona Spain.

Received for publication Oct 12, 1998; accepted for publication Jan 21, 1999.

Address for reprints: Elizabeth Zavala, MD, Surgical Intensive Care Unit, Hospital Clinic, University of Barcelona, C/Villarroel, 170.08036, Barcelona, Spain.

J Thorac Cardiovasc Surg 1999;117:1219-21

Copyright (C 1999 by Mosby, Inc.

$0022-5223 / 99 \$ 8.00+0 \quad \mathbf{1 2 / 5 4 / 9 7 3 7 6}$ and L5). The electrocardiogram showed sinus tachycardia with a repolarization disturbance. Results of laboratory tests, which included hematocrit (34\%), electrolytes, myocardiumspecific isoenzymes, and serum creatinine level, were all within reference ranges.

Initial management included the introduction of a tube into the left side of the chest to remove both air and blood (600 $\mathrm{mL}$ ) and immobilization of the femur. During these procedures the patient became hypotensive and abdominal distention developed. Ultrasonography showed intra-abdominal fluid and suggested a ruptured spleen. At the beginning of the surgical laparotomy the patient had a cardiac arrest, which was successfully managed with closed-chest cardiopulmonary resuscitation. Laparotomy confirmed massive hemoperitoneum as a result of a ruptured spleen, which was treated by splenectomy. Afterward an external pelvic fixation device was applied to the patient and her femur fracture was reduced and fixed. During the surgical procedures, hypoventilation with hypoxemia developed. A tube was placed into the right side of the chest and drained $700 \mathrm{~mL}$ blood. This drainage was persistently productive, necessitating a right thoracotomy. The patient was admitted after the operation to the surgical intensive care unit.

Acute respiratory distress syndrome developed. After hemodynamic stabilization with fluid administration and with inotropic support and appropriate management of her respiratory complication, the patient's condition improved. Twenty-four hours later, changes in the hemodynamic pattern were observed that were suggestive of left ventricular insufficiency, with a low cardiac index, a high pulmonary artery wedge pressure, a low left ventricular systolic work index, and a high systemic vascular resistance. A new electrocardiogram showed a significant deep Q wave in the inferior and 

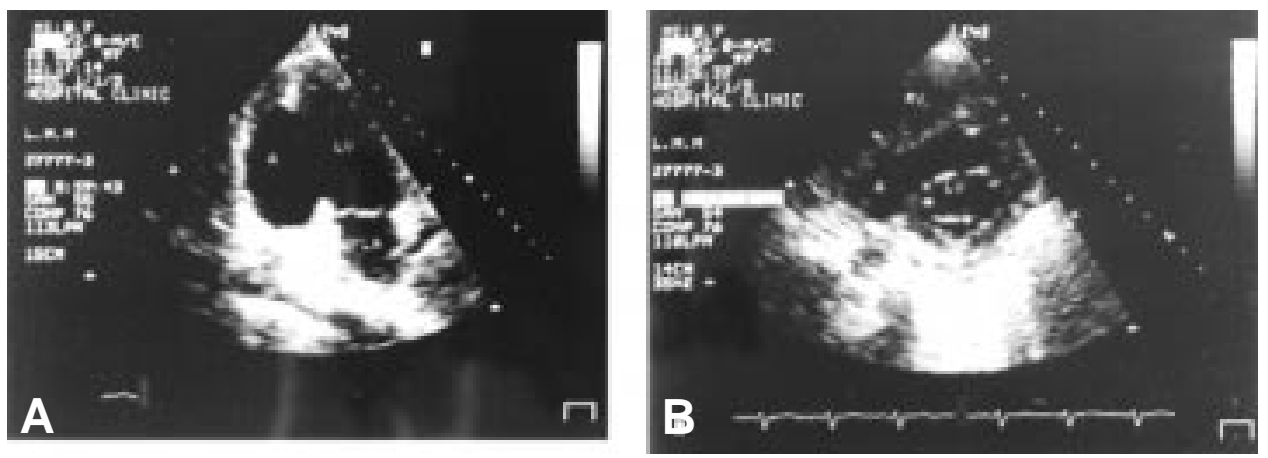

Fig 1. Transthoracic 2-dimensional echocardiography. Two-chamber (A) and short-axis (B) views show $54 \times 37$ $\mathrm{mm}$ aneurysm $(A)$ extending from inferior site of left ventricle $(L V)$ to posteroinferior site of interventricular septum. $R V$, Right ventricle.

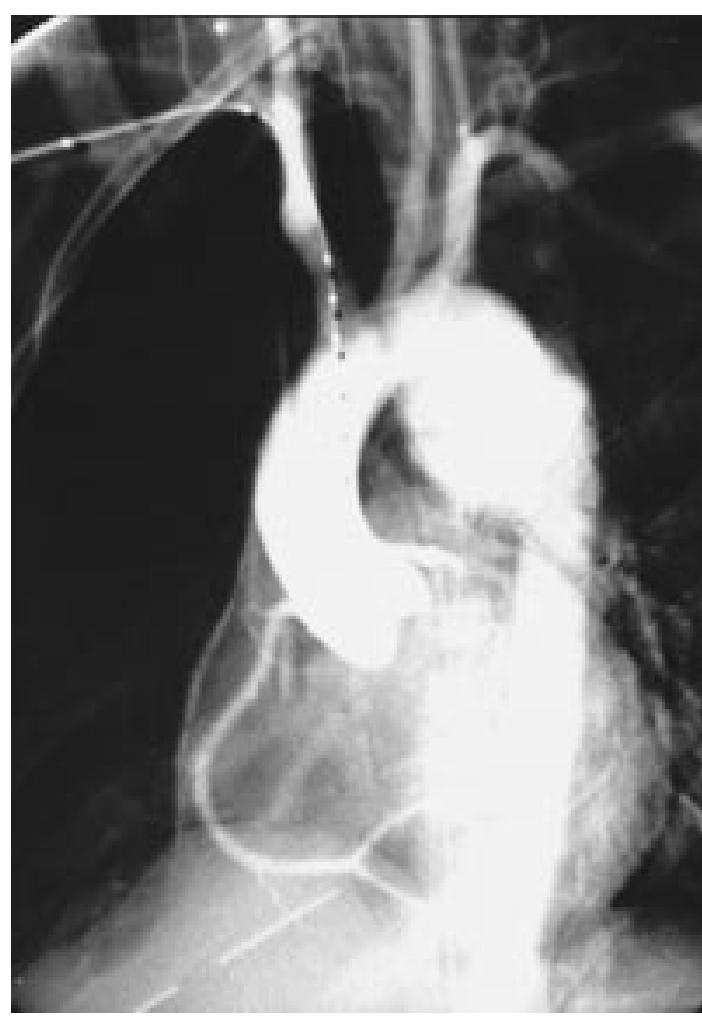

Fig 2. Supra-aortic angiography demonstrates traumatic aortic tear with pseudoaneurysm formation in vicinity of left subclavian artery. Right coronary artery patency can also be observed.

lateral leads, but the MB isoenzyme fraction of creatine kinase did not differ from the first determination on admission. The hemodynamic and electrocardiographic alterations led to the performance of a transthoracic 2-dimensional echocardiogram, which showed a $54 \times 37-\mathrm{mm}$ aneurysm extending from the inferior site of the left ventricle to the posteroinferior site of the interventricular septum (Fig 1). A sub- sequent coronary angiogram did not show coronary lesions, but the aortogram demonstrated a traumatic aortic tear with pseudoaneurysm formation just below the left subclavian artery. This finding was confirmed by supra-aortic angiography (Fig 2).

Treatment of the pseudoaneurysm was carried out by surgical isolation of the left femoral artery, transverse arteriotomy, and introduction of a $24 \times 50$-mm Vanguard endovascular stent-graft (Meadox Division, Boston Scientific Corporation, Boston, Mass) advanced under fluoroscopic guidance into the transverse thoracic pseudoaneurysm (Fig 3 ). Angiography was performed to confirm the appropriate positioning of the prosthesis, to confirm patency of both the thoracic aorta and the supra-aortic vessels, and to ensure pseudoaneurysmal exclusion. At the end of this procedure, with the prosthesis in place, laceration of the left external iliac artery occurred during removal of the introducer sheath. This complication was treated by prompt occlusion of the left common iliac artery with a balloon catheter inserted through the right axillary artery, followed by emergency surgical iliofemoral interposition with expanded polytetrafluoroethylene (W. L. Gore \& Associates, Inc, Flagstaff, Ariz).

The postoperative course was uneventful and the patient was discharged from the surgical intensive care unit 10 days after the operation. A follow-up computed tomographic angiogram performed 3 months later showed successful exclusion of the traumatic aortic tear (Fig 4). On follow-up 8 months after the operation the patient was free of symptoms and had a New York Heart Association functional classification of II with oral therapy with diuretics and angiotensinconverting enzyme inhibitors.

Discussion. The incidences of injuries to the heart and great arteries in patients with blunt chest trauma vary widely (15\% and $4 \%$, respectively). ${ }^{1}$ The combination of left ventricular aneurysm and thoracic aortic pseudoaneurysm after blunt chest injury is extremely rare in a clinical setting because the vast majority of patients with severe cardiac and aortic injuries die before reaching the hospital. ${ }^{1}$ Three mechanisms have been described for the formation of myocardial 


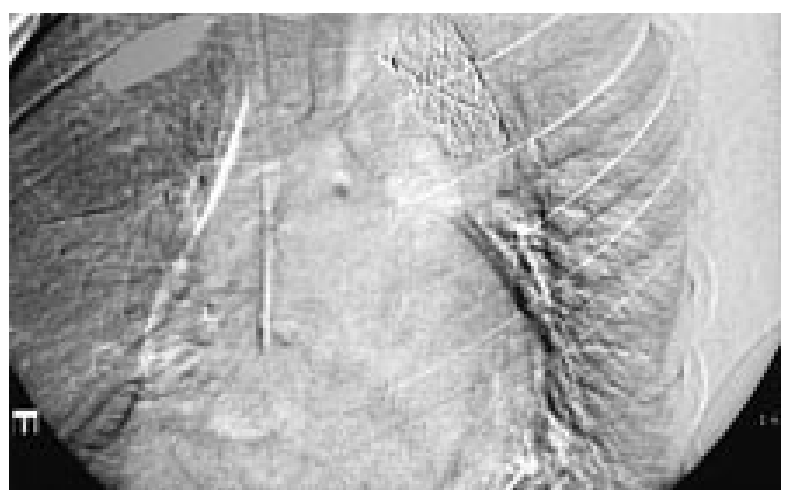

Fig 3. Chest radiograph shows endovascular prosthesis at level of thoracic aneurysm.

aneurysms: contusion, vascular lesion leading to myocardial necrosis, and intramyocardial dissection. ${ }^{2}$ On the other hand, decelerating and crushing forces to the chest are the main mechanisms resulting in injuries to the thoracic aorta. ${ }^{3}$ In our case the absence of coronary lesions suggests that the cause of the ventricular aneurysm formation was contusion with patchy areas of intramyocardial necrosis. It was the combination of an unexpected worsening of the hemodynamic parameters with a pattern of cardiac insufficiency and slight changes in the electrocardiogram that raised the suspicion of blunt cardiac injury. The ventricular aneurysm was observed with the use of thoracic echocardiography. The presence of trauma to the thoracic aorta requires the examination of the thorax by transesophageal echocardiography, a reliable method for the diagnosis of traumatic aortic injuries. ${ }^{4}$ In our patient aortic injury was not suspected and thus could be demonstrated only on the angiogram performed for the evaluation of the coronary arteries.

Pseudoaneurysms of the thoracic aorta are potentially lifethreatening lesions because they have a high tendency to rupture, and surgery is therefore mandatory. The simultaneous presence of pseudoaneurysm in the descending thoracic aorta and aneurysm of the left ventricle represents an additional difficulty in operative decision making. At present the standard treatment of the aortic tree is still surgical repair, despite its association with significant morbidity, mainly paraplegia, multiple organ failure, bleeding, left vocal cord paralysis, and nosocomial pneumonia. ${ }^{5}$ Treatment of thoracic aneurysms by endovascular stent-graft prostheses has been developed during recent years, ${ }^{6,7}$ with favorable results. This approach minimizes the risk of disruption of the intercostal blood supply to the spinal cord, with lower risk of paraplegia, and also avoids the complications associated with major surgery and cardiac bypass. This less aggressive treatment may also be a better choice for those patients with critical hemodynamic status as

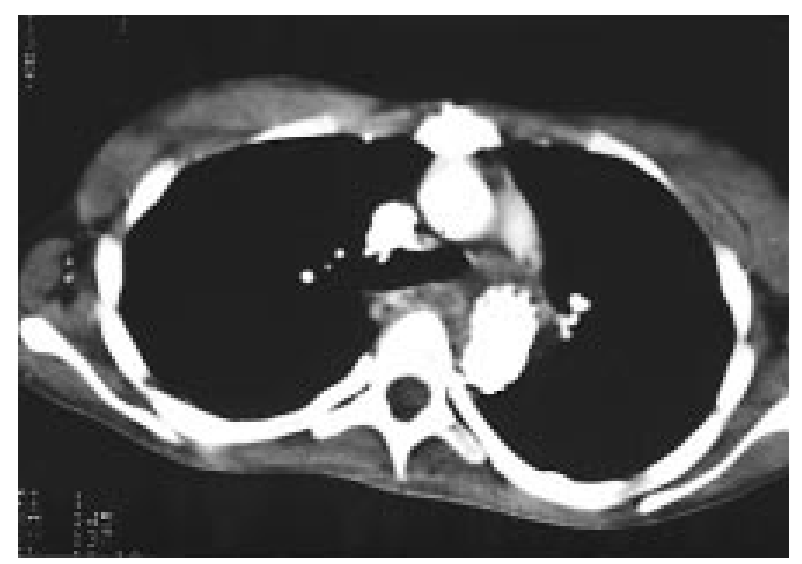

Fig 4. Computed tomographic angiographic image after 3 months shows endovascular prosthesis (stent-graft) at level of thoracic aneurysm.

a result of associated cardiac lesions. The deformity observed in the inferoseptal wall of the left ventricle in the echocardiographic images was interpreted as a real aneurysm and not as a pseudoaneurysm. On this diagnostic basis, and considering that rupture of a ventricular true aneurysm is rare beyond the first week after diagnosis, surgical repair was not performed. Ten months' echocardiographic follow-up demonstrated similar size and appearance of the aneurysm as in the first study. As far as we know, this is the first reported case in which an endovascular stent-graft was successfully used to treat a pseudoaneurysm of the thoracic aorta in a patient with traumatic aortic disruption combined with a ventricular aneurysm.

\section{REFERENCES}

1. Prétre R, Chilcott M. Blunt trauma to the heart and great vessels. N Engl J Med 1997;336:626-32.

2. Maselli D, Micalizzi E, Pizio R, Audo A, Gasperis CD. Posttraumatic left ventricular pseudoaneurysm due to intramyocardial dissecting hematoma. Ann Thorac Surg 1997;64:830-1.

3. Creasy JD, Chiles C, Routh WD, Dyer RB. Overview of traumatic injury of the thoracic aorta. Radiographics 1997;17:27-45.

4. Goarin JP, Catoire P, Jacquens Y, et al. Use of transesophageal echocardiography for diagnosis of traumatic aortic injury. Chest 1997;112:71-80.

5. Hunt JP, Baker CC, Lentz CW, et al. Thoracic aorta injuries: management and outcome of 144 patients. J Trauma 1997;40:547-56.

6. Dake MD, Miller DG, Semba CP, Mitchell RS, Walker PJ, Liddell RP. Transluminal placement of endovascular stent-grafts for the treatment of descending thoracic aortic aneurysms. N Engl J Med 1994;331:1729-34.

7. Mitchell RS, Dake MD, Semba CP, et al. Endovascular stentgraft repair of thoracic aortic aneurysms. J Thorac Cardiovasc Surg 1996;111:1054-62. 